\title{
Reforma Constitucional en Derechos Humanos y los Derechos Individuales y Colectivos de los Pueblos Indígenas en México ${ }^{1}$
}

\author{
Adria Velia González Beltrones ${ }^{2}$ \\ María Dolores Rocha Ontiveros ${ }^{3}$ \\ Guadalupe Aleida Valenzuela Miranda ${ }^{4}$
}

\begin{abstract}
Sumario: Introducción. I. Exhortos de la CEDAW a los Gobiernos y situación real en México II. Reconocimiento en lo Formal III. Etapas de la observancia del Principio de igualdad IV. Corolario
\end{abstract}

Resumen. En este documento se analizan dos casos de mujeres indígenas de Oaxaca a la luz de la Reforma Constitucional de Derechos Humanos de 2011 ,lo que muestra la urgencia de que el Estado Mexicano cumpla 1) con las obligaciones derivadas de las normas nacionales e internacionales que reconocen derechos individuales y colectivos a los pueblos indígenas tomando en cuenta su rezago y marginación histórica, para que todas y todos tengan acceso y protección de todos sus derechos humanos, sociales, económicos, culturales, civiles y políticos, y 2) con dotar a las comunidades indígenas de mecanismos de exigibilidad, para que se conviertan en una realidad en su vida cotidiana lo que implica adecuaciones a los programas educativos, a los servicios de salud, intérpretes y traductores en las dependencias administrativas y juzgados de las entidades federativas. Toda vez que mientras no se logre que la mitad de la población indígena mexicana(niñas y mujeres indígenas) tenga acceso y protección de todos sus Derechos Humanos, la práctica de la democracia y la observancia del estado de derecho, la justicia y la paz, no serán realidad en nuestro país.

Palabras clave: Derechos de las mujeres indígenas - Obligaciones del Estado Mexicano - Responsabilidades de autoridades administrativas Responsabilidades de autoridades judiciales

\footnotetext{
1 Documento presentado en Western Social Science Association WSSA's 57th Annual Conference Latin American Studies Seccion Abril 8-11, 2015 Portland, Oregon USA

${ }^{2}$ Doctora en Ciencias Políticas y Sociales por la UNAM. Profesora Investigadora de Tiempo Completo de la Universidad de Sonora adriag@sociales.uson.mx

${ }^{3}$ Doctora en Administración Pública, por la Universidad de Veracruz e Instituto Sonorense de Administración Pública ISAP, Profesora Investigadora de Tiempo Completo de la Universidad de Sonora drocha@sociales.uson.mx

${ }^{4}$ Doctora en Ciencias de la Educación por la UNED España. Profesora Investigadora de Tiempo Completo de la Universidad de Sonora avalenzuela@sociales.uson.mx
} 


\begin{abstract}
:
In this paper, we will discuss two cases of indigenous women of Oaxaca in light of the Constitutional Reform of Human Rights of 2011,which shows the urgency of the Mexican State to meet 1) with the obligations under national and international standards that recognize individual and collective rights to indigenous people taking into account their lag and historical marginalization, for that all have access and protection of all their human rights, social, economic, cultural, civil and political, and 2) giving the indigenous communities of mechanisms available to enforce, to become a reality in your daily life which means adequacies Educational programs, health services, interpreters and translators in the administrative agencies and tribunals of federal entities.

While it is not possible to get half of the Mexican Indians (girls and indigenous women) access and protection of all their human rights, the practice of democracy and the observance of the rule of law, justice and peace, would not be a reality in our country.
\end{abstract}

Key words: Human rights, individual rights of indigenous women, obligations of the Mexican State, responsibilities of the administrative authorities, responsabilities of judicial authorities

\title{
Introducción
}

En los países tanto industrializados como en desarrollo, las mujeres y las niñas, pertenecientes a pueblos indígenas o etnias padecen los efectos de prácticas tradicionales perjudiciales, cuyas raíces están en supuestos culturales ancestrales o en creencias religiosas, que en ocasiones amenazan sus vidas. De acuerdo con el INEGI, en 2010 en México por la lengua que hablan se identifican 68 pueblos indígenas.

Esta población, sea por razones históricas o por los desplazamientos que han sufrido y que los han excluido del desarrollo, padece una gran dispersión territorial. La distribución de la población por el tipo de municipios según la presencia de población indígena, a partir de los resultados del censo 2010, tiene las siguientes características generales: 
- El número de municipios indígenas, donde la concentración de población indígena es mayor al $40 \%$, asciende a 624 ,

- Ello representa el $25.4 \%$ del total de los 2,456 municipios del país.

- En ellos viven el $58.4 \%$ de la población indígena del país $(6,500,722$ habitantes). ${ }^{5}$ Comparados con la población total en estos municipios, la población indígena equivale a un $75.5 \%$.

Día tras día aparecen en los principales medios, reportajes--noticias como los siguientes:

Caso 1 Se presenta en varias comunidades indígenas de Oaxaca:

Diana(12 años) fue "entregada en matrimonio" por su familia, por los usos y costumbres del pueblo de Santa María Yucunicoco, Oaxaca a José de 21 por ocho cartones de cerveza, 20 litros de mezcal, 10 rejas de refresco y un par de gallinas. Vinculado al alto índice de migración, en Yucunicoco los matrimonios de niñas y adolescentes con hombres mayores o de su misma edad forman un círculo negativo que mantiene a la población dentro de los indicadores más altos de rezago social.

- El Instituto Nacional de Estadística y Geografía (INEGI) precisa que: la comunidad Santa María Yucunicoco ocupa

- el primer lugar con mayor número de personas mayores de 15 años que sólo cuentan con educación básica (51 por ciento de su población).

- De la misma forma, encabeza la lista con mayor número de viviendas sin drenaje, con piso de tierra y sin energía eléctrica;

- además de que la mayor parte de sus habitantes no son derechohabientes de algún servicio de salud.

Sin fuentes de empleo o actividades productivas que abran otras expectativas, las y los jóvenes no tienen a su alcance opciones para un proyecto de vida más allá que lo que la migración o el matrimonio les ofrece. Mientras que un 20 por ciento de quienes acuden a la telesecundaria decide por la primera opción, el resto opta por ser padre o madre de familia ${ }^{6}$

\footnotetext{
${ }^{5}$ FUENTE: INEGI. Censo de Población y Vivienda 2010. Población de 5 años y más que habla lengua indígena por entidad federativa y lengua según condición de habla española y sexo (Catálogo INEGI)

CIMAC noticias, Periodismo con perspectiva de género.(2014), Centro de Documentación de Comunicación e Información de la Mujer, A. C. http://www.cimacnoticias.com.mx/node/67216 Por Citlalli López Velázquez, corresponsal
} 


\section{Caso 2}

"En San Martín Itunyoso municipio Triqui ${ }^{7}$-, por si un hombre va y le gusta una mujer sin haber sido su novio, sin haberla tratado o siquiera conocerla, puede comprarla. Basta que vaya con los papás de ella para que le digan cuánto es y pueda conseguirla.En algunas familias se impone el requisito de que deben casarse con ellas y darle de comer a toda la familia el día de la boda, pero esto no siempre se cumple", algunos hombres han intentado evitar el pago por la compra de mujeres, como es el caso Mauricio quien pagó una multa de 60 mil pesos, un toro, 30 rejas de refresco, 30 cartones de cerveza, 20 litros de aguardiente y tortillas, por haberla llevado a su casa. $Y$ es que en medio de la pobreza, las familias ven en el matrimonio de las niñas la solución al problema de alimentar muchas bocas, tanto por el "intercambio" de dinero y regalos, como por liberarse de un integrante.

El gobierno del estado de Oaxaca ha fracasado en abatir esa práctica; tan sólo de enero a mayo de 2014, de los 62 mil 679 eventos obstétricos, 13 mil 811 fueron de mujeres entre 12 y 19 años de edad, (22 por ciento de madres adolescentes). Mauricio explica que en Yucunicoco, como en la mayoría de las localidades de Juxtlahuaca y otros municipios de la región triqui, las mujeres se casan o comienzan a vivir en unión libre a partir de los 12 años. "Cuando van a pedir a las mujeres tienen que ir a su casa y estar toda la noche hasta que convenza a los padres de la novia dándole de tomar cerveza, aguardiente, así hasta que casi se emborrache para que dé las respuestas. Desde ese momento las mujeres quedan apartadas y ya no pueden salir a los bailes o a la escuela. No pueden salir solas a la calle", expresa. Detalla que para él, el matrimonio sólo retrasó un poco sus planes de migración. Tiene contemplado irse en unos meses para pagar el dinero que pidió prestado para casarse. Por ser todavía menor de edad, 17 años, Reyna, esposa de Mauricio, se quedaría en casa porque sin credencial de elector no podría trabajar en los campos de cultivo en Camalú, sic Baja California. http://www.cimacnoticias.com.mx/node/67216

\section{Exhortos de la CEDAW a los Gobiernos y situación real en México}

Por casos, como los expuestos, desde hace ya más de 20 años, la Convención para la Eliminación de todas las Formas de Discriminación contra la Mujer, CEDAW por sus siglas en inglés, hizo énfasis en la necesidad de eliminar

\footnotetext{
${ }^{7}$ Ibidem
} 
todas las prácticas perjudiciales para las mujeres y las niñas, independientemente de cuan profundamente esten arraigadas en la cultura. Por eso, la CEDAW exhorta a los gobiernos a tratar de transformar no solo el derecho, sino también la cultura, a fin de lograr la igualdad y la equidad entre los sexos y entre las personas. Desde hace mas de 20 años nuestro país se comprometió a poner fin a las prácticas tradicionales que siguen perjudicando a las mujeres y niñas indígenas y sometiéndolas a estereotipos, bajo el pretexto del conformismo social, la necesidad económica o la supervivencia cultural.(Secretaría de Desarrollo Social[SEDESOL],2011:114)

Resultado de estas Convenciones --por lo menos se percibe en lo formal--la producción de legislación federal y local y/o sus modificaciones en relación a la protección de la mujer en general y a las mujeres de las etnias en particular. Asimismo se percibe en los diversos Programas derivados de la aplicación de Políticas Públicas relativas, según se muestra y se puede observar en los portales de los Institutos de las Mujeres de cada entidad así como sus homólogos municipales.

Pero, ¿que sucede en nuestra realidad?, lamentablemente continúa presentándose la condición de violencia y de minusvalía en la que viven, además de las mujeres de las etnias, las mujeres de la periferia de las grandes ciudades y las de las urbes capitalinas. En un mundo androcéntrico como en el que coexistimos, todavía se presentan prácticas como: la preferencia por los hijos varones, la dote y la violencia de género.

Preferencia por los hijos varones

- Se distingue a los niños varones quienes reciben alimentos y cuidados en condiciones preferenciales y son más estimados por sus familias y la propia comunidad.

- las niñas por el contrario, están malnutridas, no asisten a la escuela, asumen responsabilidades y trabajos domésticos muy pesados a muy temprana edad $y$

- a menudo se les obliga precozmente a contraer matrimonio con objeto de obtener la dote, o inclusive se les vende sin saber cuál es 
su suerte final, que en muchos casos se convierte en prostitución o pornografía.

\section{La dote}

- Si bien en nuestro país se ha abolido legalmente la institución de la dote,

- persisten en las comunidades indígenas su práctica y arraigo cultural.

- Van en aumento los informes acerca de esposos y suegras que asesinan o lesionan clandestinamente a las esposas compradas con dotes muy costosas,

- porque esta costumbre refuerza el estereotipo de que la mujer es propiedad de su esposo.

La Violencia de Género: Que es un patrón de conducta presente en la vida de todas las mujeres y las niñas indígenas ${ }^{8}$,

- incluye maltrato físico, sexual y psicológico,

- se debe a los estereotipos de propiedad e inferioridad de las mujeres y de superioridad de los hombres.

- del maltrato en la casa paterna, las mujeres pasan al maltrato en la casa conyugal,

- Ilegan inclusive a considerar que es natural ya que es un fenómeno común en la vida de todas las mujeres y las niñas indígenas.

La falta de equidad de género está profundamente arraigada en la vida de las niñas indígenas desde su nacimiento, o incluso antes. Las muchas prácticas violentas o discriminatorias comprometen la salud de las niñas indígenas y reducen sus oportunidades de desarrollo social y productivo. En palabras de Ulloa " no se trata de una violencia aleatoria: el factor de riesgo es ser mujer y ser indígena, agravada por su edad lo que las coloca en una situación de mayor vulnerabilidad"...

De acuerdo a la Encuesta Nacional sobre la Dinámica de las Relaciones en los Hogares ENDIREH ${ }^{9}$, de 2011, 3 de cada 5 mujeres sufren de algún tipo de violencia. Dos años después ---9 marzo, 2013---, en una entrevista para el Diario La Jornada Michoacana, la Senadora María del Rocío Pineda Gochi., expresó :

\footnotetext{
${ }^{8}$ Ulloa Ziáurriz Teresa C., "La situación de las mujeres indígenas en México", Ponencia originalmente presentada ante la Mesa de Derechos de las Mujeres Indígenas, organizada por la Federación Internacional de Derechos Humanos, Comisión Mexicana de Promoción y Defensa de los Derechos Humanos sobre la Legislación Indígena en México. México 2002, pp1-3

${ }^{9}$ INEGI Instituto Nacional de Geografía y Estadística (2013) Encuesta Nacional sobre la Dinámica de las Relaciones en los Hogares ENDIREH . Disponible en:

http://www.inegi.org.mx/est/contenidos/proyectos/encuestas/hogares/especiales/endireh/endireh20 11/default.aspx [Consultado 07/03/2015]
} 
“Las mujeres, aún víctimas de violencia y discriminación en México", en ese mismo tenor, en nota informativa de ese mismo año de Quadratin ${ }^{10}$ se observa:

"Unen esfuerzos en defensa de mujeres de Uruapan La presidenta honoraria del DIF Uruapan, señora Ana Cervantes de Macías; la senadora Rocío Pineda Gochi y la diputada federal Socorro Quintana León acordaron sumar esfuerzos en defensa de las mujeres maltratadas, al señalar que la violencia hacia las féminas es uno de los problemas que más lastiman al tejido social."

Asimismo penosamente Ulloa resume ---según se puede observar en el siguiente párrafo---, los orígenes de tal estigma :

La discriminación en la alimentación es la mayor causa de enfermedad y muerte de las niñas indígenas, entre su nacimiento y los cinco años de edad......las niñas reciben menor cantidad y calidad de alimentos que los varones. Se las amamanta por lapsos más breves, reciben menos calorías y padecen desnutrición (que a la larga las conduce a la muerte o a la discapacidad mental o física), con tasas superiores a las de los varones........ las niñas tienen menor acceso a la atención de la salud. ....... sufren además de otras prácticas y tradiciones culturales que las mantienen en riesgo constante como el matrimonio precoz, el embarazo adolescente, los frecuentes abusos sexuales y la violación, así como la prostitución y la pornografía infantil. Esas prácticas tienen efectos devastadores sobre la salud de las niñas y las colocan en situación de mayor riesgo durante toda su infancia. Además, las que sobreviven y llegan a ser adultas pueden prever que han de tener menos oportunidades de educación, empleo y sobrevivencia. En México, las familias efectúan inversiones en los hijos varones debido a que consideran que son posibles fuentes de recursos y apoyo para el hogar en el futuro y para el trabajo en el campo; las niñas, que con frecuencia se consideran cargas económicas, proporcionan no obstante en el hogar una fuerza de trabajo tan valiosa que muchas familias no pueden permitirse enviarlas a la escuela para que reciban una educación que mejoraría radicalmente su condición económica y social. $^{11}$

Y lastimosamente, los datos proporcionados, siguen vigentes, pues al parecer, lograr avances en lo social representa mayor dificultad que la inherente a los avances tecnológicos, y la situación de las mujeres de las etnias se agrava considerablemente si se hace referencia a sus derechos económicos dado que :

\footnotetext{
10 Agencia Quadratín,(2013/01/15)Unen esfuerzos en defensa de mujeres de Uruapan. Disponible en:

https://www.quadratin.com.mx/regiones/Unen-esfuerzos-en-defensa-de-mujeres-de-Uruapan/ [Consultado 07/03/2015]

${ }^{11}$ Ulloa, op.cit. p. 3
} 


\begin{abstract}
Las mujeres indígenas cumplen múltiples funciones en el trabajo productivo, pero, mayormente no remunerado, que no se refleja en la medición oficial de la actividad económica. Las mujeres indígenas carecen del todo de reconocimiento y acceso a derechos como a igual remuneración por trabajo igual, a prestaciones familiares, a la propiedad y al crédito, al derecho a ser propietarias y a heredar bienes $y$ esto se debe a prácticas tradicionales profundamente discriminatorias que siguen vigentes en forma explícita en la vida económica de las mujeres indígenas, situación que además es avalada en muchos casos por la propia legislación vigente $^{12}$.
\end{abstract}

\title{
II. Reconocimiento en lo Formal
}

En este punto cabe recalcar que no obstante que el discurso de los derechos humanos en nuestro país -a partir de las Reformas de 2001 y 2011 de nuestra Carta Magna ${ }^{13}$ - proporciona un nuevo lenguaje moral y un marco legal para resolver las demandas de los pueblos indígenas, ello se da, de nuevo en lo formal pero no en lo sustantivo, pues no obstante que en nuestro México tanto el gobierno federal como los gobiernos locales por medio de instituciones como el Instltuto Naclonal Indlgenlsta INI - Comlslón Nacional para el Desarrollo de los Pueblos Indígenas CNDPI (1948-2014),el Instituto Nacional para las Mujeres INMUJERES, sus homólogos en las entidades federativas y sus municipios, y algunos organismos de la sociedad civil o no gubernamentales realizan esfuerzos considerables tratando de revertir todas estas desigualdades, no se ha logrado a cabalidad tal propósito.

\footnotetext{
${ }^{12}$ Ibid p. 3

${ }^{13}$ de acuerdo con el artículo $2^{\circ}$ de la Constitución Política de los Estados Unidos Mexicanos (CPEUM), México es una nación que "tiene una composición pluricultural sustentada originalmente en sus pueblos indígenas". De este reconocimiento se desprende una serie de derechos para los pueblos, comunidades e individuos indígenas de México, cuya justiciabilidad es fundamental para el modelo del Estado de derecho pluricultural que perfecciona la Carta Magna. Parte de la trascendencia de la reforma constitucional en la materia radica en reconocer a los derechos humanos definidos por las fuentes jurídicas de derecho internacional como parte del sistema constitucional mexicano.

("Protocolo de Actuación para quienes Imparten Justicia en casos que involucren Derechos de Personas, Comunidades y Pueblos Indígenas" Suprema Corte de Justicia de la Nación, 2014 p.10,
} 
Por lo anterior, y reconociendo que es mucho mas, lo que nos corresponde implementar o modificar, sobre el tema de los Derechos Humanos y de las recientes Reformas Constitucionales en los diferentes rubros, de trascendental importancia para la vida de nuestro país y la convivencia armónica entre las personas tan heterogéneas que en él habitamos ---, tomando como modelo los estudios de caso expuestos en este documento, paso a delinear como propuesta, la urgencia de que en México, las condiciones de igualdad y sus formas de realización sean respetadas. De allí que, en los párrafos siguientes, proceda a enunciar las probables acciones que correspondería realizar por parte de las autoridades administrativas y jurisdiccionales para los justiciables, de conformidad con la reforma constitucional de 2011.

\section{Etapas de la observancia del Principio de igualdad}

El principio de igualdad se reconoce en el artículo 1 Constitucional cuando se dice que "todas las personas gozarán de los derechos humanos reconocidos en esta Constitución y en los tratados internacionales de los que el Estado mexicano sea parte" y mediante la prohibición expresa de la discriminación en razón "del origen étnico o nacional, el género, la edad, las discapacidades, la condición social, las condiciones de salud, las religiones, las opiniones, las preferencias sexuales, el estado civil o cualquier otra característica que atente contra la dignidad humana". Asimismo, el artículo 4 constitucional consagra que "el hombre y la mujer son iguales ante la ley".

El derecho a la igualdad ha sido reconocido como una norma de jus cogens -normas imperativas de carácter general en el derecho de fuente internacional-, puesto que es un principio fundamental sobre el que descansa todo el andamiaje jurídico del orden público nacional e internacional. En concordancia con lo anterior, la Suprema Corte de Justicia de la Nación ha determinado que el principio de igualdad se configura como uno de los valores superiores del orden jurídico, lo que 
significa que sirve de criterio básico para la producción normativa y para su posterior interpretación y aplicación.

Se ha reconocido que para alcanzar la igualdad es indispensable eliminar la discriminación que restringe el ejercicio de los derechos.

En este contexto, enseguida, mediante el análisis de los Casos de las mujeres indígenas de nuestra exposición se pasa a señalar los alcances de la igualdad, desde las tres dimensiones en que puede concebirse: formal, real o sustantiva y estructural.

\section{a) Igualdad formal}

Es la referida a la igualdad ante la ley y supone que las personas se encuentran en un mismo punto de partida respecto del goce y ejercicio de los derechos; es decir, con base en el principio de universalidad, todas las personas gozan de todos los derechos. La igualdad formal exige la eliminación de los obstáculos legales para que las personas accedan al goce y ejercicio de los derechos y postula la eliminación de fueros y privilegios .Sin embargo, la igualdad formal no es suficiente para asegurar el acceso igualitario a los derechos ${ }^{14}$

\section{Propuesta}

Dado que la igualdad formal no es suficiente para asegurar el acceso igualitario a los derechos, es necesario un sentido de la igualdad que se haga cargo de las situaciones de hecho -los usos y costumbres- por lo que se requiere en los casos a estudio y similares, la intervención del estado en las comunidades indígenas para sensibilizar a la población sobre los DDHH regulados en nuestra Constitución y la importancia de observarlos dentro de la comunidad, por medio de la educación formal (escuelas) e informal (medios de comunicación, gráficos, radiofónicos, televisivos, o talleres sobre derechos humanos) la regulación de la

\footnotetext{
${ }^{14}$ Como los Pueblos de Yucunicoco y de San Martín Itunyoso municipios Triqui de Oaxaca, que impiden a todas las mujeres de esos pueblos el pleno goce de sus derechos, como los casos expuestos, se considera a las mujeres menores o mayores de edad, incapaces de ejercer sus derechos reconocidos en la Constitución y en los Tratados Internacionales de los que México forma parte, entre ellos el derecho a decidir por si mismas el contraer matrimonio o no y el derecho de escoger con quién.
} 
institución del matrimonio, la paternidad y maternidad responsable y los derechos y obligaciones que derivan de ella etc., en sus propias lenguas, se concienticen de tales desigualdades y lo importante que será para la propia familia su erradicación. a través de platicas, talleres, entre otras actividades sobre el tema de los derechos humanos, la regulación de la institución del matrimonio, la paternidad y maternidad responsable y los derechos y obligaciones que derivan de ella, en sus centros comunitarios, apoyados por personas originarias de la etnia que han avanzado en sus estudios y desarrollo profesional además de ser testimonios en si mismas de gozar plenamente de los derechos que otorga sin distinción de persona nuestra Constitución.

\section{b) Igualdad real o sustantiva}

La igualdad sustantiva -de facto, material o real- supone la modificación de las circunstancias que impiden a las personas el ejercicio pleno de los derechos y el acceso a las oportunidades a través de medidas estructurales, legales o de política pública. En este aspecto de la igualdad, se analizan las condiciones reales en las que se encuentra una persona -por ejemplo, su posición económica y social- en los casos que nos ocupa--- integrantes de una etnia---, para saber si la norma será eficaz para permitir el efectivo goce y ejercicio de sus derechos. Para hacer efectiva la igualdad, se debe tomar en cuenta la situación de vulnerabilidad o discriminación en la que se encuentran ciertas personas por pertenecer a determinados grupos. En su caso, deberá decidirse la pertinencia de una distinción con la finalidad de atender las asimetrías en el acceso a los derechos. Por ello, es permitido, inclusive exigido, el establecimiento de acciones afirmativas $^{15}$, Las medidas de ese carácter pueden llegar a otorgar, durante un tiempo, al sector de la población de que se trate, en los casos a estudio, mujeres

\footnotetext{
${ }^{15}$ que son aquellas medidas temporales especiales de orden legislativo, administrativo, económico, de política pública, entre otras, utilizadas para reducir o eliminar las condiciones que originan 0 facilitan que se perpetúe la desigualdad y la discriminación.
} 
de los pueblos de Santa María Yucunicoco,o de Itunyoso-, un trato preferencial justificado encaminado a equilibrar la forma en que acceden a sus derechos. ${ }^{16}$

\section{c) Igualdad estructural}

La igualdad estructural se refiere no sólo y exclusivamente a la idea de no discriminación (entendida como trato no arbitrario fundado en prejuicios), sino que comprende también a un trato no segregacionista y no excluyente tendiente a consolidar la situación de grupo como marginado.

Así, en los casos de nuestra exposición, se puede observar una total ausencia de igualdad estructural, ya que el contexto social (comunidades triquis del Pueblo de Yucunicoco, o de Itunyoso, Oaxaca) juega un papel fundamental en el trato que se les da a las mujeres y niñas indígenas. Es decir, la realidad social en la que viven las mujeres de esos lugares, con actitudes ancestrales de desvalorización del papel que corresponde desempeñar a la mujer -no les permite aceptar que las mujeres al igual que los hombres tienen derecho a vivir una vida libre, (todo como consecuencia de su pertenencia a dicho grupo) mujeres triquis- históricamente sometido, por el grupo triqui masculino. Además, la desigualdad estructural también está ligada a la carencia sistemática de actitudes positivas -trasmitida de generación en generación- hacia el rol que debe desempeñar la mujer en la sociedad y del ejercicio de los derechos más elementales. La desigualdad estructural ha sido considerada por el Derecho Internacional de los Derechos Humanos al hablar sobre la discriminación sistémica $^{17}$. El Comité ha constatado periódicamente que la discriminación contra algunos grupos subsiste, es omnipresente, está fuertemente arraigada en el comportamiento y la organización de la sociedad y a menudo implica actos de discriminación indirecta o no cuestionada. "Esta discriminación sistémica puede consistir en normas legales, políticas, prácticas 0 actitudes culturales

\footnotetext{
${ }^{16}$ Por ejemplo, reservar un número determinado de puestos para los hombres y mujeres de las etnias, que contraigan matrimonio libremente.

17 Observación General No. 20 La no discriminación y los derechos económicos, sociales y culturales (artículo 2, párrafo 2 del Pacto Internacional de Derechos Económicos, Sociales y Culturales)
} 
predominantes en el sector público o privado que generan desventajas comparativas para algunos grupos y privilegios para otros."

Asimismo, éste tipo de discriminación exige medidas compensatorias y transformativas del contexto en el que se genera tal exclusión -en los casos a estudio- el ejercicio de los derechos de igualdad ante la Ley, así como el sometimiento sistemático de la mujer a las decisiones androcéntricas. Estas medidas pueden ser consideradas o implementadas por todas las autoridades en el ámbito de sus respectivas competencias, por ejemplo, en el caso que nos ocupa se debe estudiar la discriminación estructural presente en el entorno socio-cultural "comunidades triquis" y su relación con la intolerancia a aceptar que las mujeres como Diana y Reyna, esposa de Mauricio- tienen los mismos derechos que los hombres, por lo que el Estado mexicano debe emprender medidas con una vocación transformadora de dicho entorno.

No solo medidas de carácter restitutivo, o de establecimiento de acciones afirmativas, que coadyuven al cambio de actitud en relación al rol que tradicional y ancestralmente en dicha comunidad triqui como en otras de la especie le han asignado a la mujer; sino además medidas definitivas que propicien avances.

Además, dado que la igualdad, en sus tres dimensiones, es un principio que rige la producción normativa y su interpretación. Por lo tanto, las autoridades judiciales deben combatir la discriminación estructural y garantizar acceso realmente igualitario a los derechos -en el caso a estudio- ejercer Diana y la esposa de Mauricio el derecho de igualdad ante la Ley.

Por último cabe señalar que casos como los expuestos que lleguen ante las autoridades jurisdiccionales, sea por abandono de sus esposos o por falta o incumplimiento de alimentos, deberán los operadores judiciales observar los puntos 4.10 y 4.11 sobre los Derechos económicos, sociales y culturales y sobre lo indígena y sus implicaciones en un proceso jurídico, ambos del Protocolo de Actuación para quienes imparten Justicia en Casos que involucren Derechos de Personas, Comunidades y Pueblos Indígenas, además de aplicar el Protocolo para Juzgar con Perspectiva de Género, que si bien, fueron elaborados para 
auxiliar a los operadores del Poder Judicial ante los que se les presenta en sus diferentes fases, asuntos de la naturaleza precitadas, también pueden ser observadas para el mismo propósito, por toda autoridad de la administración pública (poder ejecutivo) y del poder legislativo.

\section{Corolario}

Como corolario extraído y adaptado de los Protocolos Precitados, información de los portales digitales de organismos gubernamentales y no gubernamentales y de la bibliografía consultada sobre el tema, se lista los siguientes puntos con la aspiración de que nos mueva a la reflexión y urgencia de la acción:

1. Las mujeres indígenas sostienen a una parte muy importante de la población indígena, mediante tareas remuneradas y no remuneradas que les imponen una semana de trabajo de 70 o más horas.

2. Dos terceras partes de los analfabetas mexicanos son mujeres indígenas,

3. Dado que las leyes sobre títulos de propiedad y tenencia de la tierra, así como las prácticas tradicionales, por lo general excluyen a las mujeres de la propiedad de la tierra, por lo que,

4. A las mujeres casadas, se les niega el derecho a la propiedad o a heredar y si lo permiten, le asignan el poder de administración de los bienes exclusivamente a los hombres.

5. Los obstáculos jurídicos y culturales a la propiedad y a la administración de bienes constituye una importante causa de la pobreza de la las mujeres indígenas.

6. La falta de equidad en el orden laboral y económico de México, además de la contribución voluntaria de la mujer al sostenimiento del hogar en forma remunerada o no, perpetua su condición de desigualdad e inequidad.

7. Las mujeres indígenas migrantes y las desplazadas, son doblemente discriminadas y violentadas cuando se encuentran fuera de su comunidad.

8. No obstante que por un lado, las normas que a nivel nacional e internacional reconocen derechos individuales y colectivos a los pueblos indígenas y que deben garantizar a todas y todos el acceso y protección de todos sus derechos humanos, sociales, económicos, culturales, civiles y políticos, además de dotar a las comunidades indígenas de mecanismos de exigibilidad, para que se conviertan en una realidad en su vida cotidiana lo que implica adecuaciones a los programas educativos, a los servicios de salud, intérpretes y traductores en los juzgados del Estado, etc. Esta 
adecuación cultural y el establecimiento de medidas de refuerzo para que los indígenas puedan acceder a los derechos del Estado democrático, toma en cuenta su rezago y marginación histórica y establece obligaciones de hacer para el Estado, a la fecha, en la práctica no se ha logrado cumplir a cabalidad con el compromiso contraído con la comunidad internacional al respecto.

9. Por otro lado, las normas nacionales e internacionales de derechos humanos desarrollan los alcances del derecho a la libre determinación que los pueblos indígenas tienen en atención, no a su situación de pobreza, marginación o exclusión, sino a su continuidad histórica como pueblos que existían antes de la conformación del Estado mexicano. No obstante que estos derechos son eminentemente colectivos, es decir no se ejercen por individuos; a la fecha, en la práctica tampoco se ha logrado cumplir a cabalidad con el compromiso contraído con la comunidad internacional al respecto.

10. Si no se logra que la mitad de la población indígena mexicana(niñas y mujeres indígenas) tenga acceso y protección de todos sus Derechos Humanos, la práctica de la democracia y la observancia del estado de derecho, la justicia y la paz, en nuestro país será solo aspiración, quimera o ficción.

\section{Referencias Bibliográficas y Webgrafía}

Anaya James (2013),Relator Especial sobre los derechos de los pueblos indígenas de conformidad con la resolución 15/14 del Consejo de Derechos Humanos de la ONU, Misiva de dirigida al Dr. Carlos Pérez Vázquez, Coordinador de Derechos Humanos y Asesoría de la Presidencia de la Suprema Corte de Justicia de la Nación.

Convención de Viena sobre el Derecho de los Tratados. U.N. Doc A/CONF.39/27 (1969), en vigor desde el 27 de junio de 1980. Disponible en: http://www.oas.org/legal/spanish/documentos/convencionviena.htm [Consultado 07/03/2015]

Corte Interamericana de Derechos Humanos CIDH (2003), Condición Jurídica y Derechos de los Migrantes Indocumentados. Opinión Consultiva OC-18/03 del 17 de septiembre de 2003, Serie A No. 18, párr. 101. Disponible en: http://www.corteidh.or.cr/docs/opiniones/seriea_18_esp.pdf[Consultado 07/03/2015]

Corte Interamericana de Derechos Humanos CIDH (2012), Caso Atala Riffo y Niñas vs. Chile. Sentencia de Fondo, Reparaciones y Costas de 24 de febrero de 2012, Serie C No. 239, párr. 79. Disponible en: http://www.corteidh.or.cr/docs/casos/articulos/seriec_239_esp.pdf [Consultado 07/03/2015]

Mayorga Nuvia (2014), "El reto indígena" Especial sobre Pueblos Indígenas.

Revista México Social, pp. 1 
Marcos Sylvia (2014), “Derechos Humanos y Mujeres Indígenas” Especial sobre Pueblos Indígenas. Revista México Social, pp 1

Pobreza e Igualdad, "Justicia y Género" Boletín No. 41, noviembre de 2012. Programa de Equidad de Género de la Suprema Corte de Justicia de la Nación. Disponible en: www.equidad.scjn.gob.mx/IMG/pdf/Boletin-GyJNov2012.pdf

"Protocolo de Actuación para quienes Imparten Justicia en casos que involucren Derechos de Personas, Comunidades y Pueblos Indígenas" Suprema Corte de Justicia de la Nación,2014 pp.10,

Protocolo para Juzgar con Perspectiva de Género Suprema Corte de Justicia de la Nación,2013

¿Qué es la igualdad formal y qué es la igualdad sustantiva? Programa de Equidad de Género de la Suprema Corte de Justicia de la Nación. Disponible en: www.equidad.scjn.gob.mx/spip.php?page=faq\&id_rubrique=195\#p3

Saba, Roberto, (Des)igualdad estructural. Revista Derecho y Humanidades, No. 11, Universidad de Chile, 2005. Pág. 142. Disponible en:

www.derechoyhumanidades.uchile.cl/index.php/RDH/article/viewFile/17057/17779 [Consultado 07/03/2015]

-Ulloa Ziáurriz Teresa C., "La situación de las mujeres indígenas en México", Ponencia originalmente presentada ante la Mesa de Derechos de las Mujeres Indígenas, organizada por la Federación Internacional de Derechos Humanos, Comisión Mexicana de Promoción y Defensa de los Derechos Humanos sobre la Legislación Indígena en México. México 2002, pp. 1-3

\section{Webgrafía}

Agencia Quadratín,(2013/01/15)Unen esfuerzos en defensa de mujeres de Uruapan. Disponible en: https://www.quadratin.com.mx/regiones/Unen-esfuerzos-en-defensa-de-mujeresde-Uruapan/ [Consultado 07/03/2015]

Centro de Documentación de Comunicación e Información de la Mujer, A. C. (2014/07/29), López Velázquez,C., corresponsal, Autoridades son inútiles ante los usos y costumbres, Emigrar o casarse, única opción para niñas indígenas en Oaxaca. Disponible en: http://www.cimacnoticias.com.mx/node/67216 [Consultado 07/03/2015]

Instituto Nacional de Geografía y Estadística (2013) Encuesta Nacional sobre la Dinámica de las Relaciones en los Hogares ENDIREH. Disponible en: http://www.inegi.org.mx/est/contenidos/proyectos/encuestas/hogares/especiales/e ndireh/endireh2011/default.aspx [Consultado 07/03/2015]

Secretaría de Desarrollo Social.(2011), Modelo Actualizado de Atención a Mujeres en situación de Violencia. Apegado a lo establecido en la Ley de Acceso de las Mujeres a una Vida Libre de Violencia para las Mujeres en el Estado de Chiapas. Tuxtla Gutiérrez Chiapas, México. Disponible en: http://vidasinviolencia.inmujeres.gob.mx/vidasinviolencia/sites/default/files/pdfs/sist ema_nacional/modelos/atencion/MODELO_DE_ATENCION-1.pdf [Consultado 02/02/2015] 